\title{
Progress in \\ Cardiovascular Diseases
}

VOL. XXV, NO. 5

MARCH/APRIL 1983

\section{Symposium on Computer Applications to Cardiology \\ Introduction}

Janice M. Jenkins, Ph.D., Guest Editor

$\mathbf{T}$ HE IMPACT of computers in the field of medicine has been a dramatic one. The computer has emerged as a major technical tool in the acquisition, analysis, interpretation, and storage of medical data both in the research laboratory and in the clinical setting. Cardiology represents a discipline where some of the earliest computer applications occurred, particularly in the area of electrocardiography. With the advent of integrated circuit technology computing power has expanded dramatically as the physical size of computers has shrunk. Microprocessors no larger than a postage stamp are an integral part of many medical devices, and larger computer systems are interfaced to a variety of clinical instruments. As the use of these computerized devices increases the physician will find it essential to know and understand their operation. This symposium examines eight areas in cardiology in which the computer has become a significant feature, ranging from electrocardiography and cardiovascular imaging through drug infusion.

The paper, "Automated Elcctrocardiography and Arrhythmia Monitoring," apprises us that the electrocardiogram was the first physiologic signal to be processed and analyzed by digital computer. The technique dates back to 1957 when Hubert Pipberger developed a system for automatic recognition of electrocardiographic waves by computer. Early attempts at computerderived measurement of the waveforms progressed to pattern recognition methods for discrimination and classification, and eventually to diagnostic interpretation of the electrocardiogram (ECG). In the first section a historical review is presented of the evolutionary stages of computer interpretation of the diagnostic ECG. The reader is acquainted with various methods employed for signal processing, techniques for waveform and contour analysis, and algorithms for diagnostic interpretation. Commercial systems which evolved from those developed in research settings are described and distinctive features are pointed out.

The article presents a chronicle of early attempts at rhythm analysis (Section III) and the continuing difficulties associated with this problem. Pattern recognition and measurement techniques applied with success to analysis of the QRS complex perform poorly in $\mathbf{P}$-wave detection due to the small amplitude of the signal and its low frequency content. Computer-based arrhythmia monitors for coronary care were a natural outgrowth of the interpretive system but ineffective $\mathrm{P}$-wave recognition continues to be the major limiting factor in rhythm analysis. The development of a miniaturized esophageal clcctrode for computer-detection of $\mathrm{P}$-waves pro-

\footnotetext{
From the Department of Electrical and Computer Engineering, The University of Michigan, Ann Arbor, Mich.

Reprint requests should be addressed to Janice M. Jenkins, Ph.D., Department of Electrical and Computer Engineering. The University of Michigan, Ann Arbor, Mich. 48109

(c) 1982 by Grune \& Stratton, Inc.

0033-0620/83/2505-0001\$01.00/0
} 
vided a dramatically improved registration of atrial activity and enabled for the first time automated analysis of complex arrhythmias. The two-lead system which recognizes $\mathbf{P}$-waves on the esophageal signal, QRS complexes on the surface lead, and computes PP, RR, and PR intervals is described in Section $V$ of the paper.

Computer methods for ambulatory monitoring, high-speed playback and analysis are discussed in Section VI. The problems associated with evaluation and testing of computer ECG systems remain a major consideration and are presented in Section VII. Initial attempts at evaluation of the diagnostic ECG have been reported but no consistent scheme has emerged which can be applied in general for comparative purposes. Proposals for the development of a test library with clinical documentation from nonECG sources have been advanced, but the complexity of the task has served to inhibit its implementation. A promising move in this direction is the creation of an annotated data base for arrhythmia monitors developed under the auspices of the American Heart Association for purposes of testing automated detection systems.

The introduction of computers into clinical electrocardiography has not resulted in any widespread improvement of diagnostic accuracy or dramatically altered the delivery of medical care, but the technique of computer analysis and storage of large numbers of ECGs within a single system provides a powerful tool for epidemiologic studies and possible early detection of cardiovascular disease. Technologic advances include remote acquisition and telephonic transmission of ECGs to a central computer facility and advanced electrocardiographs with internally contained microprocessors which instantly deliver a diagnostic report at the patient site.

One paper in our series deals with mapping of the electrical activation of the epicardial and endocardial surfaces of the heart, a procedure which has become an important technique in clinical cardiology and also in basic research. There have been striking advances in the use of mapping in the surgical treatment of drugresistant arrhythmias, and mapping during surgery has provided increased knowledge of car- diac activation patterns during sinus rhythm, artificial pacing, and spontaneous arrhythmias. The authors, Smith and Ideker, review the computer techniques which have arisen and contributed to the advancement of mapping technology. Early traditional methods with limited data acquisition and analysis capabilities have been replaced by modern systems for recording, manipulation, and data display. Their paper, "Computer Techniques for Epicardial and Endocardial Mapping," covers all aspects of the mapping operation from the application of electrodes to the graphical presentation of results. Two major phases of cardiac mapping are discussed: data acquisition and data analysis. The authors describe both computer and noncomputer methods for data acquisition, but point out that computer methods are essential for analysis purposes because of the vast quantity of data produced by the mapping technique.

Another review in this symposium presents "Cardiac Catheterization and Angiographic Analysis Computer Applications." From the middle 1960s efforts have been made to utilize computer systems for the analysis of data from cardiac catheterization. This paper presents an overview of work done in this area by numerous investigators and describes in detail the features of a computerized cardiac catheterization system developed at the University of Alabama in Birmingham Medical Center. The system has been in clinical use since 1973 and currently supports two adult catheterization laboratories. Four ana$\log$ signals, three hemodynamic pressure measurements, and the electrocardiogram can be monitored simultaneously. The completely automated system computes all hemodynamic and electrocardiographic parameters, determines measurement of cardiac output by the thermodilution technique, stores calculated data, and provides reports of the procedure to the physician. An additional program which is not part of the routine pressure analysis system is capable of computing isovolumic indices of muscle function. These algorithms are described by Zissermann, et al. in Section II of the paper.

In Section III, an angiographic analysis system is described in which on-line data acquisition and quantification of left ventricular (LV) 
dimensions is computer-controlled. Instrumentation in the angiographic data laboratory for digitizing LV silhouettes and computer processing techniques for quality control and report generation are presented in detail. Stroke volume and ejection fraction measurements are determined by computer and results of volumetric analysis and segmental wall motion analysis are provided in the form of a clinical report.

A paper in this series on Computer Applications in Cardiology describes the "Construction and Interpretation of Body Surface Maps." The authors, Barr and Spach, examine the procedure of deriving maps of cardiac electrophysiologic activity at the body surface. Such maps, which portray the variation in space of potentials generated by the heart, are constructed for a single instant in time, and a sequence of such maps depicts the spatial distribution during the cardiac cycle. The fact that spatially distributed sources within the heart produce corresponding surface maps instant by instant is the basis for the interpretation of surface maps. The principal advantage of examining potential variation in space, rather than in time, is that the relationship between the spatially distributed electrical sources within the heart and potential distributions produced on the body surfaces for any instant in time can be considered independent of the relationship that exists at any other instant in time. In particular, there is a more or less direct relationship between the spatial distribution of the cardiac sources and the observed spatial distribution of potentials on the body surface.

The authors describe the measurements necessary for a body surface map and discuss the problems of establishing baseline voltages for each of the multiple electrodes, and of precise time alignment between leads. For better visualization of the voltage pattern, maps are generally presented in the form of contour patterns with the lines on the map corresponding to isopotentials. Barr and Spach utilize 24 measurements to construct a map and demonstrate that this number of measurements is sufficient to determine the entire map within a tolerance close to the noise level. The relative accuracy of maps constructed from 150 versus 24 measurements is determined both by theoretical and operational methods. Interpretation of surface maps by evaluation of the features of the waveform relative to past experience has not become widely used due to the fact that it is more difficult technically to obtain maps; thus only small numbers are available for analysis and the precise format varies from place to place. Interpretation of maps can be accomplished by mathematical estimation. Epicardial potentials computed inversely from body surface potentials are compared to measured epicardial surface maps in dog studies and the inverse maps appear to be sufficiently good approximations to the measured maps. The authors conclude that the widespread use of mapping as a clinical or research tool will await development of standard commercial devices for map processing, so that potential users are not required to begin by taking on the construction of a surface mapping device. They predict the advent of such systems and a continued increase in the use of mapping procedures.

One review in this symposium is entitled "Computerized Cardiovascular Imaging" authored by Bulawa and Meyers. The application of computer technology to image analysis in cardiology is surveyed and current techniques are described for automated extraction of data from imaging modalities common in cardiovascular laboratories. The derivation of physiologically relevant information from an image through computer processing can be described as a two-step process: object characterization and parameter extraction. The purpose of object characterization is to retrieve from the image those features which are needed for subsequent analysis. Bulawa and Meyers discuss digital methods for image enhancement, computer determined object characterization, and calculation and display of object functional parameters. Computer applications to radiological studies for cardiac volume and ejection fraction determination, left ventricular wall motion analysis, and arteriography are presented in detail. Digital image processing is emerging as an increasingly important factor in the evolution of radiological image acquisition and analysis and it has become an integral element in the technologies that are being developed and explored. The digital image offers an alternative to film as the 
primary recording medium for radiography, and interactive image processing methods can be applied which make real time analysis a reasonable prospect for the near future.

The paper written by Jelliffe presents a review of "Computer-Controlled Administration of Cardiovascular Drugs." The author concentrates on those aspects of cardiovascular drug administration which actually have employed a computer in the process of planning, monitoring, or adjusting the delivery of the pharmaceutical agent. Two general types of application have developed: one which achieves automatic control of a system in a data-rich situation, and another which operates in a data-poor situation whereby the response has been sampled only infrequently. In data-rich situations, computer-controlled infusion strategies have been developed for control of blood pressure, cardiac arrhythmias, and blood sugar. Pharmacokinetic models have been advanced which determine the desired concentration of drug and are used in simulation of closed-loop adaptive control systems for drug delivery. Open-loop (nonfeedback) procedures are almost entirely dependent upon pharmacokinetic models and one of the earliest clinical applications of such a method was for digitalis therapy. These concepts have been incorporated into a computer program for developing loading and maintenance dosage regimens of glycosides adjusted to body weight and renal function. The program has been in use since 1973 by community hospitals over internationally accessible time-sharing facilities, and results have illustrated the utility of applying pharmacokinetic concepts in clinical situations in a quantitative manner.

A computer-assisted regimen for achieving and maintaining therapeutic serum levels of lidocaine was developed which overcame the therapeutic hiatus of low concentrations during the first 10 minutes of therapy typically seen with conventional regimens. Applications of feedback procedures in open-loop control of drug therapy, in which the patient's clinical response is compared to the model's computed body concentrations and therapeutic adjustments resulted in more accurately achieved serum levels than those achieved by physicians armed with similar phar- macokinetic knowledge. The development of optimal strategies for monitoring serum concentration to yield the greatest accuracy in the computation of each patient's pharmacokinetic values is described by Jelliffe. However, he points out that closed-loop strategies consider only how to use information that has already been obtained and make no provision for the fact that future data will also be obtained. A truly optimal closed-loop strategy would compute an optimal balance between the past information already available and that to be obtained from other data in the future. This promises to be the next stage in computer-controlled drug infusion.

The paper in this symposium which examines the role of "Computing in Echocardiography," provides insight into difficulties and limitations surrounding computer interpretation of ultrasound data. The author, Gibson, describes the predictable adoption by echocardiographers of image enhancement techniques applied earlier to other modalities, but reminds us that mechanisms underlying image degradation must be clearly understood before algorithms can be developed to reverse them. Greyscale manipulation, smoothing, and integration of information from several beats are methods which have been borrowed from other imaging systems and applied to echocardiographic images. The first application of computers in the echocardiographic field was the analysis of $\mathrm{M}$-mode records in which routine measurements were made automatically, and later, analysis was done of cardiac motion throughout the cardiac cycle. Although this information was the same as that available from direct manual measurements, automation increased the speed and convenience with which it could be extracted.

Two-dimensional echocardiography with its comprehensive display of left ventricular cavity size, shape, and wall motion was a logical candidate for the next stage in the application of computer techniques. The promise of computerderived quantitative measurements of ventricular volume, wall thickness, and regional wall motion suggested an appealing new feature that could be added to an already valuable clinical tool. Early techniques required the manual outlining by an operator of endo- and epicardial 
surfaces for subsequent computer digitization and measurement, but more recent methods employ automated methods of boundary recognition. Two distinct problems exist with this technique: the location of a pictorial boundary on the image, and determining the relation of this pictorial boundary to an anatomic one. The latter problem is much more complex since the relation between the two depends closely on ill-understood mechanisms underlying ultrasound image formation; thus even the best edge detection is imprecise.

The ability to gain complex information about cardiac anatomy and motion requires a corresponding complexity in the methods used to its display. Gibson turns to the works of early military cartographers and a nineteenth century geographer for solutions to the problem of display of three-dimensional information. The writer suggests the most satisfactory means of demonstrating complex anatomy is likely to be a stereoscopic approach citing a number of most compelling images still extant in photographic atlases of anatomy produced at the turn of the century. Other display methods are described as well.

$\Lambda$ major section of the work is dedicated to the theme of tissue characterization which refers to the possibility of using echocardiography to gain information about tissues beyond simply their position and motion. Unlike other fields in which diagnostic ultrasound is used, relatively little effort has been made to characterize the ultrasonic properties of cardiac tissues themselves. Nevertheless, the idea has proved a stimulating one to cardiologists in view of its possible clinical significance. Gibson suggests that the detection of collagen, the relationship of ultrasonic attenuation to myocardial ischemia, and textural analysis for identification of myocardial infarction comprise new areas which might be fruitful to explore.

The final paper in this symposium is entitled "Computer Analysis of Cardiac Radionuclide Data," and treats the relatively young specialty of nuclear cardiology. The authors, Froelich. Thrall, et al., have chosen to review selected areas of nuclear cardiology which are in the process of greatest evolution and assuming more important roles. These areas require the application of quantitative techniques to the evaluation of nuclear cardiologic data and are thus dependent upon computational resources and developments.

The first application of computer technology to radionuclide ventriculography was acquisition of gated end-diastolic and end-systolic frames into computer memory a frame at a time. Manual tracing of ventricular contours was done after which area-length formulas were used for calculating volumes and ejection fraction. Newer computer methods bypass the more laborious geometric calculations and derive ejection fraction from the net ventricular count rate of the intravascular tracer. This automated procedure results in more reproducible and more accurate measurements than hand analysis and has served to move the technique from the laboratory into the clinical setting. Other important breakthroughs in nuclear cardiology have been the development of new computer data acquisition techniques for gated list mode ventriculography and multigated frame mode ventriculography.

Following a brief historical review Froelich presents a complete description of equipment required for nuclear cardiology procedures and discusses various hardware components which can be interfaced to a gamma camera. Functional descriptions are given of current software techniques found in commercial systems and a section is devoted to a description of Fourier phase analysis as a method for demonstrating subtle regional differences in cardiac contractility. Image processing methods employed in quantitative myocardial perfusion are reported and promising results as well as limitations are presented.

The final section deals with tomographic imaging in which serial slices through the long axis of the heart are obtained from multiple projections, thus providing a means of threedimensional reconstruction and measurement of the distribution of radionuclides in the body in a full three-dimensional fashion. Tomographic imaging provides a means of determining not only ventricular chamber volumes but also the size of infarcted or ischemic tissue. With the refinement of tomographic imaging and the 
development of new radiopharmaceuticals it may soon be possible to measure regional myocardial blood flow and metabolic rates noninvasively.

These reviews provide the clinician with some insight into those areas where computers have played a significant role. Commercial systems are described and research systems still under development are presented. Each of the authors has provided an extensive bibliography which should serve the interested reader with a comprehensive road map should the pathway prove inviting. 